\title{
Correction to: Study on Aging Effect of Adhesion Strength Between Polyimide Film and Copper Layer
}

\author{
Seok-Bon Koo ${ }^{1} \cdot$ Chang-Myeon Lee $^{1} \cdot$ Sang-Jun Kwon $^{2} \cdot$ Jun-Mi Jeon $^{1} \cdot$ Jin-young Hur $^{1} \cdot$ Hong-Kee Lee $^{1}$ (i)
}

Published online: 14 March 2019

(c) The Korean Institute of Metals and Materials 2019

\section{Correction to: \\ Metals and Materials International (2019) 25:117-126 \\ https://doi.org/10.1007/s12540-018-0167-7}

Unfortunately, the acknowledgements were missing in the original version of this article. The information is given below:

Acknowledgements This work was supported by the Advanced Technology Center Program funded By the Ministry of Trade, Industry and Energy (11051962) and the Technology Development Program to Solve Climate Changes of the National Research Foundation (NRF) funded by the Ministry of Science, ICT \& Future Planning (NRF-2018M1A2A2063336).

The original article can be found online at https://doi.org/10.1007/ s12540-018-0167-7.

Hong-Kee Lee

schwein@kitech.re.kr

1 Surface Technology R\&D Group, Korea Institute of Industrial Technology, Incheon 21999, Republic of Korea

2 Advanced Analysis Center, Korea Institute of Science and Technology, Seoul 02792, Republic of Korea 